\title{
QALYs in psychiatric care?
}
GREg WiLkINSON, Senior Lecturer and Director, Academic Subdepartment of Psycho- logical Medicine, University of Wales College of Medicine, North Wales Hospital, Denbigh, Clwyd; Caroline Croft-JefFreys, Research Worker, General Practice Research Unit, Institute of Psychiatry, London; H. KREKORIAN, Senior Registrar, in Psychiatry Bristol General Hospital; S. McLeES, Research Officer; and IAN FalloON, Consultant Physician, Buckingham Mental Health Service, Buckingham

In the form of economic evaluation known as costutility analysis, the consequences of health programmes are measured in time units adjusted by health utility weights (i.e., states of health valued relative to one another in terms of notional quality). The outcome of health programmes can then be expressed in terms of increased (or decreased) life expectancy and increased (or decreased) quality-oflife. This provides a device for evaluating health programmes that extend life at the expense of undesirable effects, or, that produce reduced morbidity rather than reduced mortality.

\section{$Q A L Y s$}

Over the past few years there has been growing interest in the application of Quality Adjusted Life Years (QALYs) to the assessment of health programmes. The advantages of QALYs are that they can provide an improvement on crude measures of outcome such as survival rates and a means for comparison across differing health care programmes the common aims of which are to maintain and/or improve health. Moreover, by comparing costs per QALY, health programme planners can compare the relative priority of disparate claims on scarce health resources.

This preliminary study examines the application of the QALYs approach to the field of mental health, using a clinical sample of people with long-term psychiatric disorders in whom a measure of qualityof-life was available at intervals over one year.

\section{The study}

Setting

The study took place in Buckingham, a small but rapidly growing English market town, and the surrounding rural area, and is part of a larger project evaluating mental health care provided by the Buckingham Mental Health Service. This is a comprehensive community-based service providing athome care delivery mainly by general practitioners and nurse therapists supported by primary care teams and mental health specialists. The therapeutic approach is empirical, and efforts are made to involve carers and mobilise community resources when possible. The local mental hospital is over 20 miles distant, and there is a low rate of referral to it from Buckingham.

In the Buckingham Mental Health Service the mental health professional doing the 'hands on' work receives extensive support to be able to do this with the maximum efficiency. This key worker is usually but not always a nurse therapist. Psychotropic drug management is carried out by GPs on the advice of a psychiatrist. All nurses can do reliable PSEs and deliver a full range of psychosocial interventions, all carried out under the expert guidance of psychiatrist, psychologist, social worker, and occupational therapist.

\section{Subjects}

A search was made through the Buckingham Mental Health Service records for people with functional psychiatric disorders of more than one year's duration. In addition, all the local general practitioners and members of the mental health teams were asked to suggest the names of such people known to them.

\section{Research instrument}

A comprehensive structured interview schedule was developed for a project evaluating the outcome of chronic functional psychiatric disorder over two years. All subjects, and when possible a carer, were interviewed at entry using the Present State Examination, and three-monthly ratings were made by two independent research workers of a range of sociodemographic, clinical, social, family and economic measures, and patient contacts with members of the primary care and mental health care teams were recorded. In addition, nurse therapists' regular quantitative assessments of the subject's clinical condition were recorded. 
This report is restricted to findings from the first year of the study and focuses on three measures: the Clinical Global Impressions scale as a measure of severity of clinical condition; the Charing Cross health indicator as a measure of quality-of-life; and the number of health contacts made by subjects over the period.

\section{Clinical Global Impressions (CGI)}

The Clinical Global Impressions (CGI) scale is a widely used six-point scale designed to measure current severity of mental illness (1 - not ill at all; 2 borderline mentally ill; 3 -mildly ill; 4 -moderately ill; 5-severely ill; 6-among the most severely ill of patients).

\section{The Charing Cross health indicator (CH-X) as a measure of quality-of-life}

The Charing Cross health indicator $(\mathrm{CH}-\mathrm{X})$ is one of the best known methods for expressing quality-of-life data (Rosser \& Watts, 1972; Rosser \& Kind, 1978). It is a two-dimensional descriptive scale listing eight levels of disability (from "no disability" to "unconscious") and four levels of distress (from "no distress" to "severe distress"), developed by discussion with small groups of doctors and other health professionals. Thus, people can be assigned ratings for disability and for distress, which place them within an $8 \times 4$ matrix as having one of 29 possible qualityof-life states (unconsciousness is a state of disability that is assumed to preclude distress).

During the development of the instrument, relative weights were derived for each of these 29 states on the basis of material collected in interviews with hospital doctors, nurses, healthy volunteers, and people with medical and psychiatric disorders (Rosser \& Watts, 1978; Benson, 1978). The weights produced for each state were then transformed mathematically so that death received a score of zero and good health, with no disability and no distress, received a score of one.

\section{Measuring QALYs}

The change in QALYs which result from a treatment programme is found by finding the change in the discounted present value of the remaining years of a person's life, valuing each year at its score on a quality-of-life measure. Thus, if a treatment programme changes life expectancy from one year at a quality-of-life of 0.9 (disability level 5-unable to undertake any paid employment, or to continue any education; old people confined to home except for escorted outings and short walks and unable to do shopping; housewives able to perform only a few simple tasks; distress level 3-moderate) to two years at a quality-of-life of 0.995 (distress level 1 -none; disability level 2 -slight social disability), and the discount rate is $5 \%$, the gain in QALYs is 1.04025 .
Clearly, however, a health intervention can produce an increase in QALYs even if life expectancy becomes shorter, just as it can also do so by producing an extension of life at lower quality.

\section{Basis of estimated costs}

Average GP visit costs and salaries were at 19881989 levels: GPs ( $£ 8.30$ per visit); practice nurses ( $£ 9.85$ per $30 \mathrm{~min}$.); psychiatrists ( $£ 24.95$ per hour); CPNs ( $£ 9.60$ per $30 \mathrm{~min}$.); and social workers ( $£ 9.85$ per $30 \mathrm{~min}$.).

However, the estimated costs of teamwork have been ignored for this preliminary analysis, which are intended to be illustrative and to represent an underestimate of actual costs. We estimate that the costs incurred by teamwork for each case are around $£ 30$ per hour, reflecting all aspects of training, supervision, team assessment and overheads.

\section{Findings}

\section{Sample characteristics}

Over one year 14 men and 24 women were assessed. At entry to the study they were all clinically stable. Their diagnoses were: schizophrenia (15: 9 men and 6 women); affective disorder (13: 3 men and 10 women); and neurosis (predominantly anxiety and obsessive-compulsive disorders) (10: 2 men and 8 women).

\section{Correlations between the CH-X and CGI}

In order to have a measure of its validity, the $\mathrm{CH}-\mathrm{X}$ was correlated with the CGI at entry and 3, 6, 9, 12 months, and the corresponding Spearman correlation coefficients obtained were $0.71,0.56,0.61$, 0.57 , and 0.76 , respectively.

\section{Correlations between repeated CH-X measures}

Spearman correlation coefficients were calculated for repeated $\mathrm{CH}-\mathrm{X}$ measures (bearing in mind that treatment was directed towards improving mental health) at $0 / 3,3 / 6,9 / 12$ and $0 / 12$ months, and these were $0.65,0.87,0.82,0.72$, and 0.48 , respectively.

\section{Differences in mean CH-X and CGI measures over 12} months

There was no significant difference in mean $\mathrm{CH}-\mathrm{X}$ score over the 12 months, but there was a significant difference in mean CGI ratings over the 12 months, indicating improvement with treatment.

\section{Mean change in $\mathrm{CH}-\mathrm{X}$ in difierent diagnostic groups}

As Table I shows, those with schizophrenia had the largest change in mean $\mathrm{CH}-\mathrm{X}$, followed by those with 
TABLE I

Clinical condition and mean (sd) $\mathrm{CH}-\mathrm{X}$ health indicator

\begin{tabular}{lllll}
\hline & All & Schizophrenia & $\begin{array}{c}\text { Affective } \\
\text { disorder }\end{array}$ & $\begin{array}{c}\text { Neurotic } \\
\text { disorder }\end{array}$ \\
\hline Month & & & & \\
0 & $0.94(0.08)$ & $0.92(0.09)$ & $0.94(0.08)$ & $0.97(0.03)$ \\
3 & $0.95(0.07)$ & $0.91(0.09)$ & $0.98(0.02)$ & $0.98(0.04)$ \\
6 & $0.97(0.05)$ & $0.95(0.07)$ & $0.97(0.03)$ & $0.99(0.01)$ \\
9 & $0.96(0.05)$ & $0.95(0.03)$ & $0.96(0.08)$ & $0.99(0.02)$ \\
12 & $0.96(0.05)$ & $0.95(0.02)$ & $0.96(0.08)$ & $0.97(0.04)$ \\
\hline
\end{tabular}

affective disorder. Those with neurotic disorder showed least change in mean $\mathrm{CH}-\mathrm{X}$.

\section{Estimated cost of change in $\mathrm{CH}-\mathrm{X}$}

Table II (a) shows the median estimated costs of care provided by the health and social services by diagnostic group. As can be seen, people with schizophrenia cost most and the overall median cost was $£ 184.85$ (range $£ 9.6$ to $£ 1024.4$ ).

The median estimated cost by professional group (ignoring costs of teamwork) is shown in Table II (b).

\section{Correlation between change in CH-X from 0-12 months}

The Spearman correlation coefficient between the cost incurred by each patient and the respective change in mean $\mathrm{CH}-\mathrm{X}$ between months 0 and 12 for each patients was -0.163 . These coefficients for schizophrenia, affective disorder and neurotic disorder were $-0.2952 ;-0.245$; and -0.030 ; respectively.

\section{Comment}

The measure of quality-of-life, $\mathrm{CH}$-X, correlated moderately highly with a corresponding clinical measure, providing a measure of concurrent criterion validity in the area of mental health, and repeated $\mathrm{CH}$ $\mathrm{X}$ measures showed evidence of stability, which would be expected in a measure of change used with a sample of people with long-term disorders.

As expected, there were relatively small changes in the mean CH-X and CGI measures over the 12 months, again in keeping with the long-term disorders found in this sample, and the low rate of acute recurrence within the duration of the investigation. It should also be noted that patients' clinical status had been stabilised prior to entry in the study and that, therefore, the main focus of treatment was on the prevention of further episodes of mental ill-health and in improving their social functioning.
TABLE II

Median costs of care

\begin{tabular}{lll} 
(a) By diagnostic group & & \\
& Median & Range \\
\hline Schizophrenia & $£ 188$ & $£ 10-708$ \\
Affective disorders & $£ 192$ & $£ 16-1024$ \\
Neurotic disorders & $£ 130$ & $£ 33-322$ \\
Total & $£ 185$ & $£ 10-1024$ \\
\hline
\end{tabular}

(b) By professional group*

\begin{tabular}{lclc} 
& Median & Range & No contact \\
\hline Nurse therapists & $£ 139$ & $£ 0-499$ & 5 \\
GPs & $£ 8$ & $£ 0-116$ & 17 \\
Practice nurse & $£ 0$ & $£ 0-345$ & 34 \\
Psychiatrists & $£ 0$ & $£ 0-200$ & 29 \\
Social workers & $£ 0$ & $£ 0-1024$ & 35 \\
Prescriptions & $£ 0$ & $£ 0-26$ & 21 \\
\hline
\end{tabular}

* No contacts by psychologist or occupational therapist recorded.

The difference in QALYs between entry and 12 months was also small, as was the median estimated cost of providing care - which was usually provided by the nurse therapist and the GP, albeit with the backing of teamwork. Simple arithmetic showed that the estimated costs per QALY provided were lowest in people with schizophrenia (around $£ 6,000$ ), higher in those with affective disorders (around $£ 10,000$ ), and highest in those with neurotic disorders (around $£ 25,000$ ).

A question might arise about whether resources were used to best effect, since there was little correlation between change in QALYs and estimated costs. It could be argued that the benefits of current interventions would be apparent at some future date, and this will be assessed at five years. 


\section{The QALYs approach}

The apparent simplicity and comprehensiveness of the QALY approach has much to commend it. But QALYs have also generated much controversy, with criticisms falling into two main categories, ethical and practical.

From an ethical point of view, treatment planning based on any form of economic assessment has been condemned as inevitably favouring people whose age or disease confers the prospect of longer or better quality survival. Old and seriously or chronically ill patients, it is argued, will be placed in double jeopardy, discriminated against in resource allocation decisions by reason of their poor recovery prospects and their short life expectancy.

As a basis for clinical resource allocation, QALY. based judgements imply a shift of emphasis, taking priority away from those who suffer most, and giving it to those who would benefit most from treatment. In this regard we note that the Charing Cross health indicator incorporates the social judgement that a small increase in health at the bottom of the scale may be more valuable in terms of quality-of-life than a larger gain for a more healthy patient.

Practical criticisms of current methods of calculating QALYs are more damaging. Quality-of-life measurement is still in a primitive state: there are large areas of medicine, not least psychiatry, which are arguably incapable of being characterised reliably and validly by a two dimensional index.

In fact, psychiatrists have traditionally regarded the social as well as the clinical outcomes of mental health programmes as important. Cost-benefit analyses have attempted to measure the success of programmes in terms of social adjustment criteria, such as finding work, being able to live independently and avoiding crime. Because of the lack of differences in life expectancy, criteria loosely classifiable as quality-of-life judgements are the major measure of success for psychiatric treatment.

In principle, therefore, it should be a relatively easy task to translate these more detailed judgements into health indicator scores and so produce QALYs comparisons between competing psychiatric programmes and between psychiatric and medical/surgical programmes. However, there are two problems. First, although an improvement on comparing health programmes exclusively in terms of number of lives saved, the QALYs approach still emphasises the extension of life. So mental health programmes, which rarely claim to increase life expectancy, will have to produce large, long-term increases in qualityof-life if they are not to seem 'bad value' compared to some of their medical/surgical competitors. This clearly depends on the nature of the disorders considered, since such chronic medical conditions as diabetes mellitus, arthritis, chest and neurological disorders present similar therapeutic difficulties to those found with chronic mental disorders.

Donaldson et al (1988) voice another objection to using quality-of-life judgements based on the $\mathrm{CH}$-X in evaluating long-term care. While their criticisms refer to long-term geriatric care, they would be likely to apply to any health care programme involving longterm residential care. In essence, they found that the $\mathrm{CH}-\mathrm{X}$ index was insensitive to changes in health which were detected by other, programme specific, indexes. The dimensions of disability and distress were found to be irrelevant to the health states of the patients in their study. We also question the relevance of distress in the context of chronic mental disability.

This problem is very likely to occur in rating the quality-of-life of long-term psychiatric patients, and suggests either that the $\mathrm{CH}-\mathrm{X}$ needs to be adapted considerably before it can be used as a valid indicator of health care performance across programmes, or, as Donaldson et al suggest, that QALYs should be assessed using programme specific quality-of-life measures, with pre-assigned budgets for broad categories of care: acute care, long-term care, community care and primary care.

If this view is adopted, programme specific QALYs will remain as powerful tools for resource allocation at programme level. It is important that psychiatrists use their expertise in dealing with quality-of-life issues to make sure that psychiatric care benefits from this.

\section{Conclusion}

The QALYs approach has attracted criticism as a concept for the assessment of outcomes in health care and as a tool for health planners. Psychiatry has much to gain from the development of an objective and accurate standard for comparing its work with that of other medical specialities. QALYs have still to prove that they can fill that role.

\section{Acknowledgements}

This work was supported by research grants to Drs Wilkinson and Falloon from the Mental Health Foundation and the DoH.

\section{References}

BENSON, T. J. R. (1978) Classification of disability and distress by ward nurses: a reliability study. International Journal of Epidemiology, 7, 359-361.

Donaldson, C., Atkinson, A., Bond, J. \& Wright, K. (1988) Should QALYs be programme-specific? Journal of Health Economics, 14, 229-256.

ROSSER, R. M. \& WATTS, V. C. (1972) The measurement of hospital output. International Journal of Epidemiology, 1 , 361-368.

\& KIND, P. (1978) A scale of valuation of states of illness: is there a social consensus? International Journal of Epidemiology, 7, 347-357. 